\title{
Optical Property Model for Cirrus Clouds Based on Airborne Multi-Angle Polarization Observations
}

\author{
Yi Wang ${ }^{1, *(\mathbb{D})}$, Ping Yang ${ }^{1}$, Michael D. King ${ }^{1,2}{ }^{\mathbb{C}}$ and Bryan A. Baum ${ }^{3} \mathbb{C}$ \\ 1 Department of Atmospheric Sciences, Texas A\&M University, College Station, TX 77843, USA; \\ pyang@tamu.edu (P.Y.); michael.king@lasp.colorado.edu (M.D.K.) \\ 2 Laboratory for Atmospheric and Space Physics, University of Colorado, Boulder, CO 80309, USA \\ 3 Science and Technology Corporation (STC), Madison, WI 53705, USA; baum@stcnet.com \\ * Correspondence: yiwang_atmo@tamu.edu
}

check for updates

Citation: Wang, Y.; Yang, P.; King, M.D.; Baum, B.A. Optical Property Model for Cirrus Clouds Based on Airborne Multi-Angle Polarization Observations. Remote Sens. 2021, 13, 2754. https://doi.org/10.3390/ rs13142754

Academic Editor: Anthony J. Baran

Received: 8 June 2021

Accepted: 9 July 2021

Published: 13 July 2021

Publisher's Note: MDPI stays neutral with regard to jurisdictional claims in published maps and institutional affiliations.

Copyright: (c) 2021 by the authors. Licensee MDPI, Basel, Switzerland. This article is an open access article distributed under the terms and conditions of the Creative Commons Attribution (CC BY) license (https:// creativecommons.org/licenses/by/ $4.0 /)$.

\begin{abstract}
We present an improved remote sensing technique to infer an optimal habit/shape model for ice particles in cirrus clouds using multi-angle polarimetric measurements at $865 \mathrm{~nm}$ made by the Airborne Multi-angle SpectroPolarimeter Imager (AirMSPI) instrument. The common method of ice model inference is based on intensity (total reflectivity) measurements, which is generally not applicable to optically thin ice clouds (i.e., cirrus clouds) where single scattering dominates. The new approach is able to infer an ice model in clouds with optical thicknesses smaller than 5 . The improvement is made by first assuming the optical thickness retrieved using total reflectivity. Subsequently, the polarized reflectivity is calculated based on look-up tables generated from simulated polarized reflectances computed for cirrus clouds in conjunction with eight ice particle models. The ice particle model that leads to the closest fit to the measurements is regarded as the optimal ice particle model. Additionally, an alternative method is applied that does not consider polarized reflectivity. These two methods are applied to a data sample as a proof-of-concept study where AirMSPI observed a single cirrus layer. In this case study, the hexagonal column aggregate model works for most pixels both with and without considering polarized reflectivities. The retrieval cost function is high when the camera pairs with large zenith angles are included in the retrievals. This result suggests that further studies will be necessary to have a better understanding of all eight selected ice particle models at scattering angles smaller than $100^{\circ}$.
\end{abstract}

Keywords: ice clouds; cirrus clouds; polarization; multi-angular camera; particle shape; optical property model; retrieval

\section{Introduction}

The selection of an appropriate ice particle habit model and subsequent generation of the corresponding bulk single-scattering properties are critical to the implementation of remote sensing cirrus cloud property retrievals and, subsequently, to the assessment of the cirrus radiative forcing effect [1-4]. In our view, a bulk ice particle habit model should lead to consistent optical thickness and effective particle size retrievals from measurements across different scattering viewing geometries (i.e., viewing zenith angle and viewing azimuth angles). Towards this goal of developing a model that leads to greater consistency, satellite or airborne multi-angle imaging sensors, including the POLarization and Directionality of the Earth's Reflectances (POLDER) [5] and Multi-angle Imaging SpectroRadiometer (MISR) instruments [6], as well as similar airborne instruments, provide unique observations. These sensors use fixed cameras to view a given pixel at multiple angles along the satellite or airplane path. This sampling configuration provides a valuable opportunity to evaluate the reconcilability between ice particle models and measurements at multiple scattering viewing geometries over a wide range of scattering angles. The off-nadir cameras from multi-angle imaging sensors could capture the escaped radiation 
from the side of the clouds and then improve the estimation of cloud radiative effects by retrieving more accurate cloud optical properties [7].

Based on these unique directional viewing characteristics from multi-angular observations, retrieval techniques using total reflectivity from multi-angular observations have been implemented to validate ice habit models and surface morphology such as roughening [8-11]. Meanwhile, polarimeter measurements from multi-angular observations have been applied to infer ice particle habits [12-17]. The advantage is that polarimetric reflectivities are able to carry more information about cloud properties than total reflectivities alone by being sensitive to elements of the scattering matrix besides the P11 element. In employing this method, the assumption is generally made of the cloud having a constant optical thickness of 5 or larger for the generation of look-up tables (LUTs) for use in the retrieval process. The reason for this is that polarimetric reflectivities saturate at an optical thickness of approximately 5 [18] (also see Section 2.2); beyond which multiple scattering swamps the signal. This treatment clearly distinguishes ice particle habit signals for optically thick ice clouds but may not work well for ice clouds with optical thicknesses lower than the polarization saturation threshold. Since the typical optical thickness of cirrus is smaller than 3 , it is necessary to improve the conventional retrieval method that only considers optically thick ice clouds.

The primary goal of this paper is to demonstrate an algorithm to determine the most representative ice particle model for cirrus using multi-angular polarimetric reflectivity data that overcomes the constraints on the optical thickness. Furthermore, a proof-ofconcept study is presented. The data used in this study are obtained from the Airborne Multi-angle SpectroPolarimeter Imager (AirMSPI) [19]. This study also assesses camera selection in a multi-angular retrieval technique. AirMSPI provides a set of cameras that are aligned to observe a given pixel with fixed viewing angles. More details about the cameras are in Section 3.1. AirMSPI is similar in concept to MISR, which also has a set of fixed cameras aligned to observe a given pixel both in the forward and backward directions along the satellite track. However, the viewing geometries of each camera are not the same with different multi-angular imaging satellites. Several studies indicate that viewing geometries may influence cloud retrievals due to various factors, such as potential cloud 3D effects at higher angles and variable sensitivity of the ice model to viewing geometries $[7,20,21]$. While the cameras provide a wealth of information, cloud retrievals may be more consistent if the cameras are limited to those closer to nadir to limit the 3D effects. Therefore, it is necessary to understand the impact of selecting cameras on retrievals.

The remaining part of the paper is organized as follows: Section 2 describes ice particle models and the retrieval algorithm. In Section 3.1, we introduce the airborne polarimetric measurements used as a proof-of-concept study. Section 3.2 describes optimal ice models inferred when selecting different combinations of cameras and presents analyses of the effects of camera selection on optimal ice models, followed by a discussion in Section 4 . We finally present a summary and conclusions in Section 5.

\section{Methodology}

\subsection{Ice Habit Models}

Four ice particle habits were chosen in this study, including a bullet rosette, an aggregate of 10 plates, a solid hexagonal column, and an aggregate of 8 hexagonal columns. Details of ice particle habits were defined in Yang et al. (2013) [22]. For each ice particle habit, two ice particle models with different single-scattering properties are computed, one assuming a smooth-faceted particle $\left(\sigma^{2}=0\right)$ and the other for a severely roughened particle $\left(\sigma^{2}=0.5\right)$, where $\sigma$ is a parameter that specifies the degree of surface roughness [23] for light scattering computations based on the geometric optics method. The addition of roughness to the ice particle surface results in a featureless phase function in which the halo peaks are reduced. Several studies [2,11] demonstrated that rough ice particle models are able to achieve spectral consistency in the retrieved ice cloud optical thickness. Note that a roughness factor of $\sigma^{2}=0.5$ is used for operational MODIS (Moderate Resolution 
Imaging Spectroradiometer) Collection 6 (MC6) products [24], and the roughened 8 hexagonal column aggregate ice habit in this study is the same as in the MC6 ice model. Bulk scattering properties for each model are computed by assuming a Gamma particle size distribution with an effective variance of 0.1 and an effective radius of $30 \mu \mathrm{m}$ based on a typical ice particle radius and particle size distribution. For nonspherical ice particles, the effective radius $\left(r_{e f f}\right)$ is defined as

$$
r_{e f f}=\frac{3}{4} \times \frac{V}{A},
$$

where $V$ and $A$ represent the integrated volume and projected cross-sectional area of an ensemble particle [25]. In addition, three liquid water cloud particle models are used in this study to discriminate the cloud phase in the data. The three water models are computed with the Lorenz-Mie theory with respect to three different effective radii $\left(r_{\text {eff }}=4 \mu \mathrm{m}, 8 \mu \mathrm{m}\right.$, and $16 \mu \mathrm{m}$ ) with an effective variance of 0.1 . The nonzero scattering phase matrix elements (P11 and P12/P11) of the 11 cloud particle models ( 8 ice models and 3 water models) in this study are shown in Figure 1. The 865-nm channel was selected, because this channel is less affected by a varying the ice particle effective radius and also has weak ice absorption [26]. The conservative scattering channels (e.g., visible bands and the channel centered at $865 \mathrm{~nm}$ ) contain information that is primarily dependent on the optical thickness. This is a theoretical foundation of the very popular bi-spectral shortwave technique [26]. The details for ice clouds are provided in Yang et al. (2018) [25] and will not be duplicated here.

\subsection{Algorithm}

The reflectivity ( $R$; also named total reflectivity) is defined as

$$
R\left(\mu, \mu_{0}, \varphi, \varphi_{0}\right)=\frac{\pi \times d^{2} \times I\left(\mu, \mu_{0}, \varphi, \varphi_{0}\right)}{\mu_{0} \times E_{0}},
$$

where $I\left(\mu, \mu_{0}, \varphi, \varphi_{0}\right)$ is the radiance, $E_{0}$ is the solar irradiance at 1 astronomical unit (AU), and $d$ is the Earth-Sun distance relative to 1 AU. $\varphi$ and $\varphi_{0}$ are the viewing azimuthal angle and solar azimuthal angle. The factors $\mu$ and $\mu_{0}$ are the cosines of the viewing zenith angle and solar zenith angle, respectively.

In a similar manner, the polarized reflectivity $(L)$ is defined as

$$
L\left(\mu, \mu_{0}, \varphi, \varphi_{0}\right)=\frac{\pi \times d^{2} \times \sqrt{Q\left(\mu, \mu_{0}, \varphi, \varphi_{0}\right)^{2}+U\left(\mu, \mu_{0}, \varphi, \varphi_{0}\right)^{2}}}{\mu_{0} \times E_{0}},
$$

where $Q$ and $U$ are the second and third Stokes parameters, respectively.

In radiative transfer theory, total reflectivity depends on optical thickness. However, the polarized reflectivity is no longer sensitive to optical thickness when $\tau>5$, i.e., the signal reaches saturation [18]. Figure 2 shows the total reflectivity and polarized reflectivity as a function of optical thickness, corresponding to the typical scattering geometry of the AirMSPI nadir camera (000N; see Section 3.1) applied here. The total reflectivity increases with $\tau$ in Figure 2a, but in Figure 2b, the polarized reflectivity increases with optical thickness $(\tau)$ until $\tau=2$ and then decreases. This feature indicates that the polarized reflectivity does not monotonically increase with the increase of cloud optical thickness but depends on the scattering geometry. After $\tau>5$, the polarized reflectivity has no obvious variations. The polarized reflectivity shows significant differences among the ice models when $\tau>5$, and many studies use this feature to investigate ice particle habits $[13,16,17]$. Since the polarized reflectivity changes with $\tau$ when $\tau<5$, it is not appropriate to assume a constant optical thickness for cirrus clouds. The approach adopted in this study is that the computed optical thickness for both $\tau<5$ and $\tau \geq 5$ is updated with the optical thickness inferred from the total reflectivity. A similar treatment is implemented 
by van Diedenhoven et al. (2012) [15] to infer the aspect ratio and the degree of the surface roughness of ice particles for $\tau<5$.
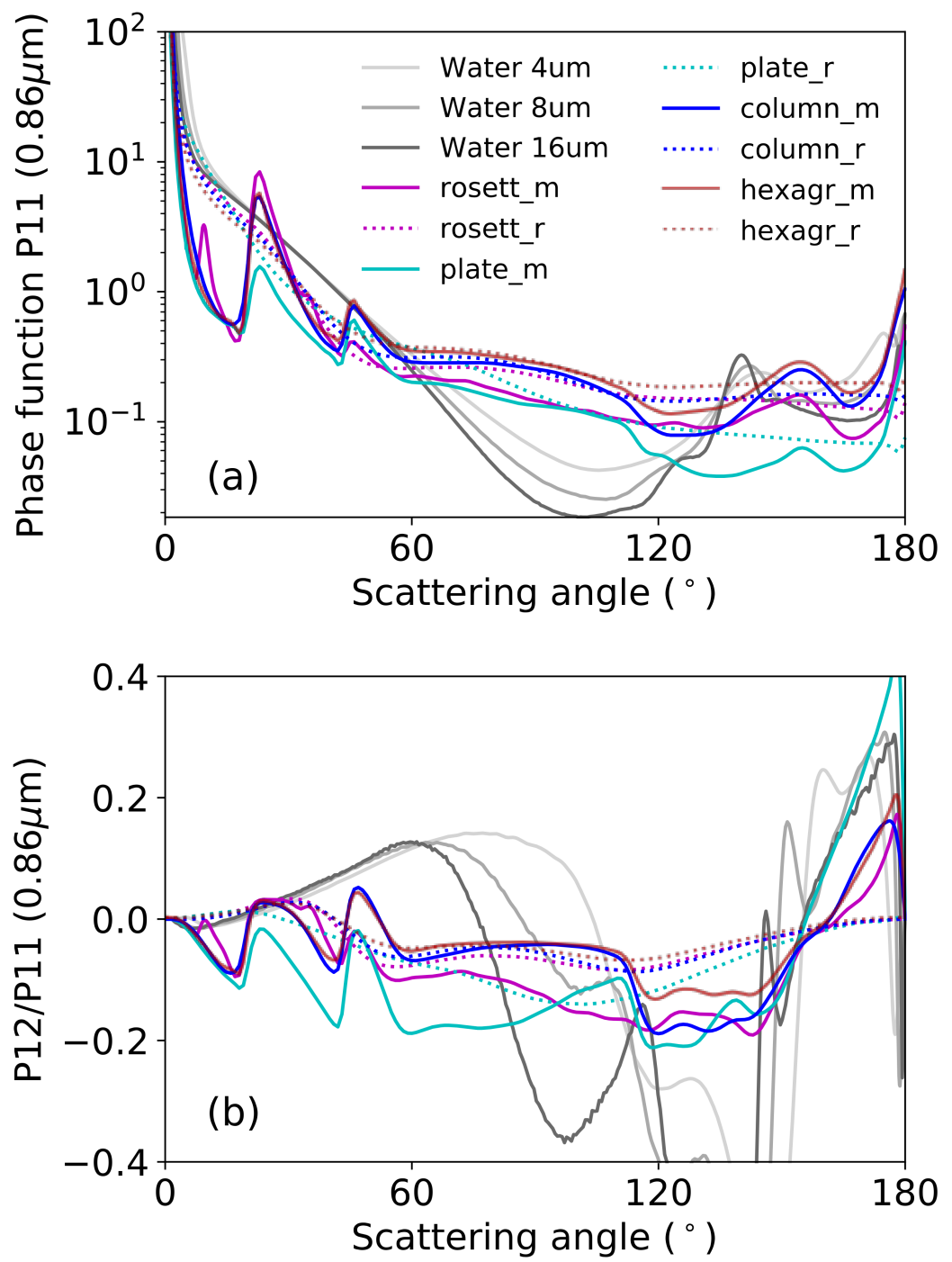

Figure 1. The (a) phase function and (b) P12/P11 phase matrix elements as functions of the scattering angle for 8 ice particle models and 3 liquid water models with different effective radii $\left(r_{e f f}=4 \mu \mathrm{m}\right.$, $8 \mu \mathrm{m}$, and $16 \mu \mathrm{m})$ at $865 \mathrm{~nm}$ used in this study. The 8 ice particle models are 4 ice particle habits, which are bullet rosette (rosette in the figure), 10 plates aggregate (plate), a solid hexagonal column (column), and 8 hexagonal columns aggregate (hexagr) with smooth surface $(\mathrm{m})$ and roughened surface (r) particles computed separately for each habit.

Figure 3 shows the flow chart for inferring an optimal ice particle model in this study using two retrieval methods. Look-up tables (LUTs) are computed for each cloud model by using an adding-doubling radiative transfer model with polarization capability [27]. This retrieval system assumes a single homogenous cloud layer and considers atmospheric molecular scattering but neglects aerosols. The ocean surface reflection is computed based on a rough ocean surface model [28]. Given the scattering geometry (i.e., solar zenith angle, viewing zenith angle, and relative azimuth angle) for each camera, the total reflectivity and polarized reflectivity as a function of optical thickness are calculated and stored in each LUT. Due to the one-to-one relationship between optical thickness and total reflectivity, the optical thickness in every viewed pixel is retrieved using the measured total reflectivity from each camera. By assuming the retrieved optical thickness, the polarized reflectivity is computed unambiguously for each camera using the LUTs. 

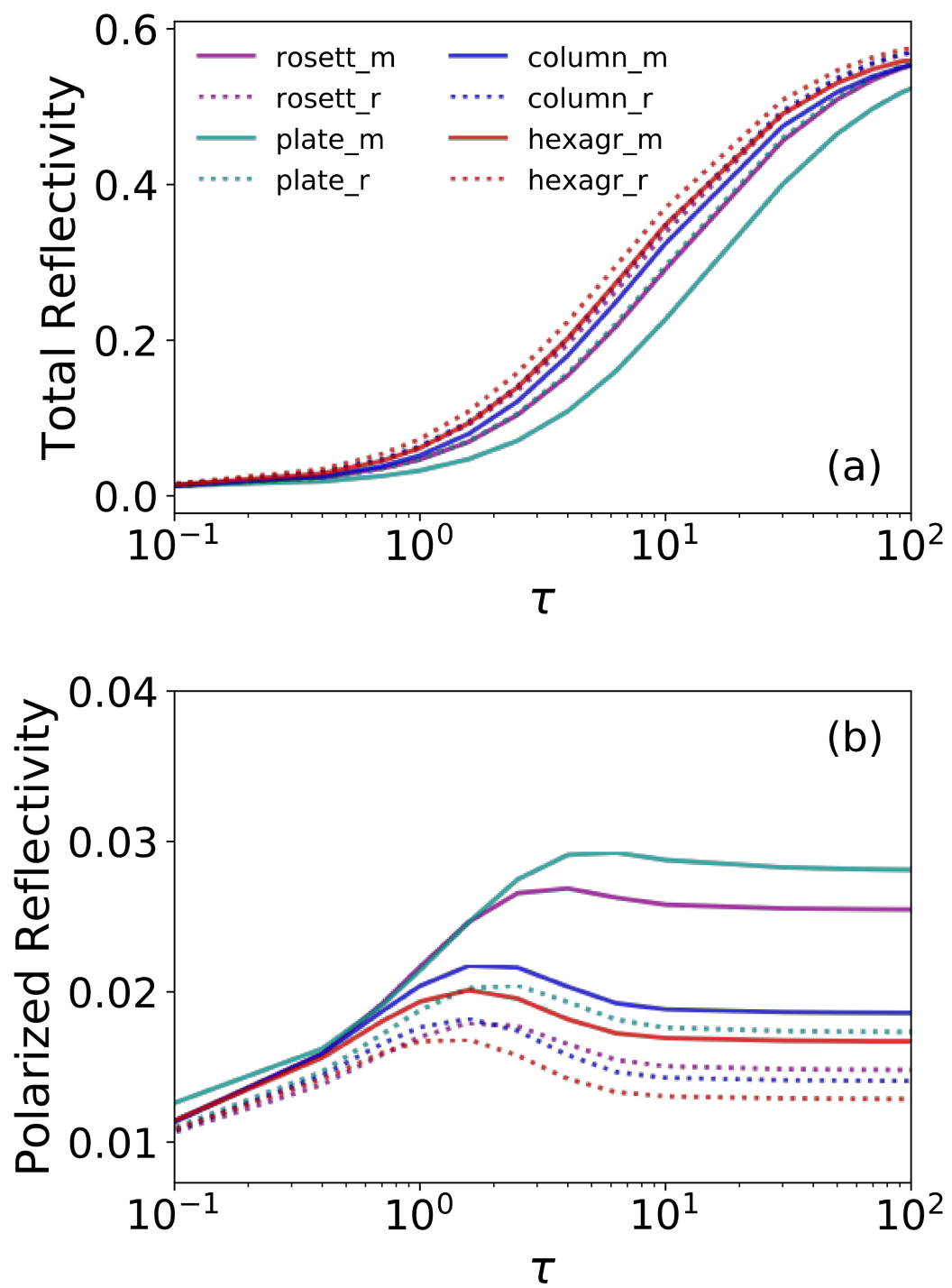

Figure 2. The (a) total reflectivity and (b) polarized reflectivity as functions of cloud optical thickness for the ice particle models used in this study. The geometry angles (solar zenith angle of $51.5^{\circ}$, viewing zenith angle of $0^{\circ}$, and relative azimuthal angle of $\left.130^{\circ}\right)$ of the AirMSPI nadir camera $(000 \mathrm{~N})$ in the chosen case study are assumed here.

Furthermore, the optimal ice particle shape is inferred using both total reflectivity and polarized reflectivity measurements. The core idea of evaluating an ice model is that the retrieved optical thickness when assuming an appropriate ice model should be the same or nearly invariant at all scattering angles. Therefore, the optimal model inferred using total reflectivity is defined as the model that provides the minimum value of the standard deviation of optical thickness retrieved from all selected cameras $\left(s_{\text {total }}^{2}\right)$ :

$$
s_{\text {total }, i}^{2}=\sqrt{\frac{1}{N} \sum_{j=1}^{N}\left(\tau_{i}-\bar{\tau}_{i}\right)^{2}},
$$

where $i$ is the selected ice particle model, $j$ is the index of the camera, and $N$ is the number of selected cameras in each pixel. The averaged value of optical thickness $(\tau)$ in each pixel is $\bar{\tau}$. A similar method is developed by Doutriaux-Boucher et al. (2000) [8] using the spherical albedo difference method. The spherical albedo difference is the spherical albedo value minus the averaged spherical albedo value of all selected cameras. Here, we apply the optical thickness difference instead of the spherical albedo difference value to see more clearly the optical thickness distributions over the range of scattering angles, since our goal 
is to develop a methodology that works from optically thin to optically thick ice clouds. Similarly, for polarized reflectivity, the optimal ice particle model is the model with the lowest relative root mean squared difference $\left(s_{p o l}^{2}\right)$ :

$$
s_{p o l, i}^{2}=\sqrt{\frac{1}{N} \sum_{j=1}^{N}\left(L_{s, i}-L_{m}\right)^{2}},
$$

where subscript $s$ indicates the simulation, and subscript $m$ indicates the measurement.

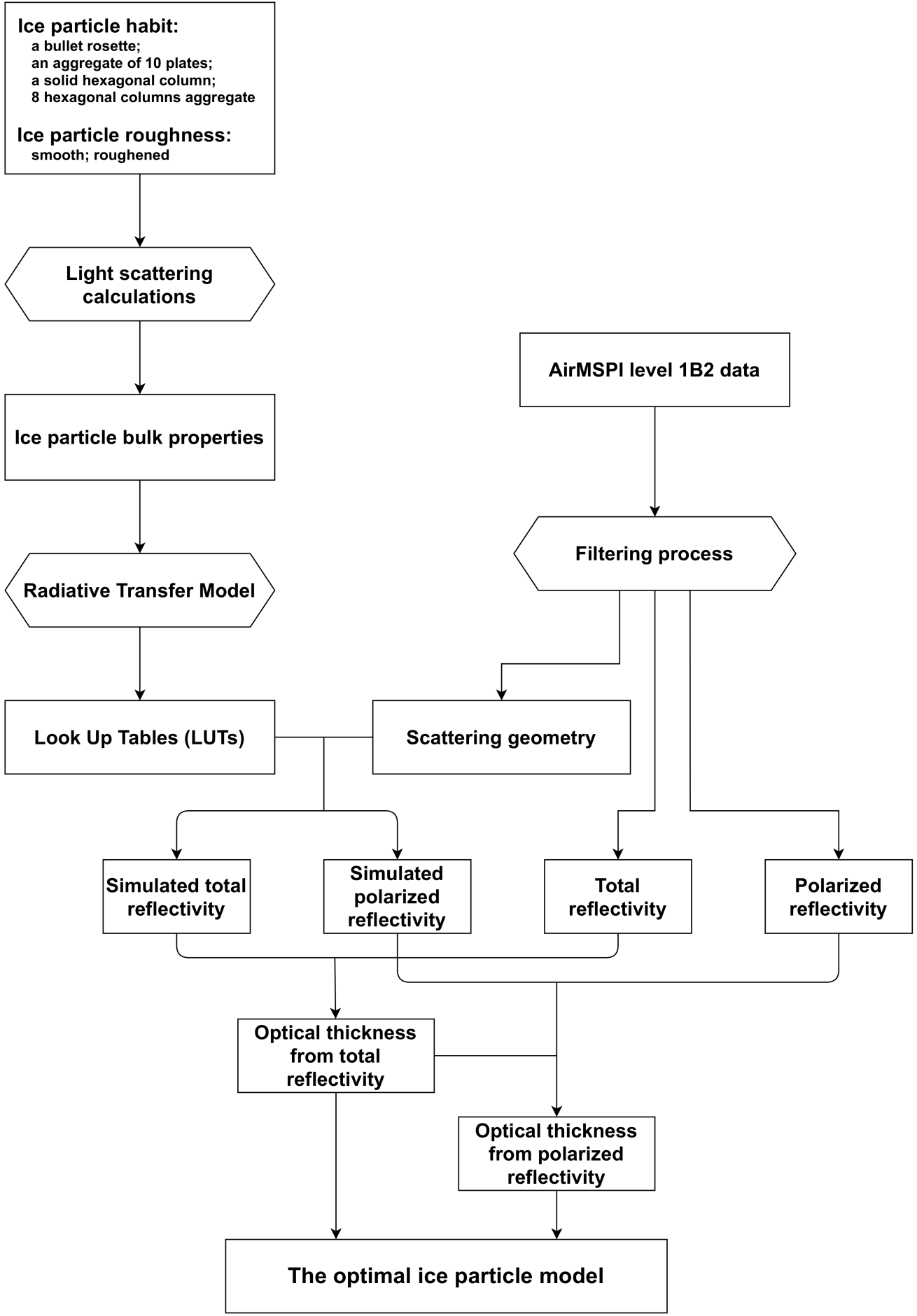

Figure 3. Flow chart for inferring the optimal ice particle models of the cirrus clouds in this study. 
Since the cloud thermodynamic phase information is not available in the dataset involved in this study, we infer the cloud phase and remove the water cloud pixels. Specifically, each pixel is retrieved by both ice and liquid water models. If the best-fit model of the pixel is from a liquid water model, that pixel is identified as a liquid water cloud and is removed. To avoid potential complications due to ocean surface reflections, a pixel is removed if its optical thickness based on the nadir camera is less than 0.1 .

\section{Case Study}

\subsection{AirMSPI Observations}

The data used in this proof-of-concept study are from AirMSPI Level 1B2 products. The AirMSPI instrument is fully described in Diner et al. (2013) [19], but briefly, it is a push broom camera deployed on NASA's ER-2 high-altitude research aircraft. The camera provides radiometric and polarimetric observations in the ultraviolet, visible, and near-infrared channels. The instrument uses two measuring modes to acquire data: step-and-stare and sweep. In this study, we only use the data from the step-and-stare mode, which provides measurements at nine viewing angles for each pixel with 10-m spatial resolution. In the step-and-stare mode, the camera is pointed at the same target at multiple viewing angles step by step. The nine directional images are 661F, 589F, 478F, 290F, 000N (camera at nadir), 291A, 478A, 589A, and 661A. The naming convention provides the viewing angle, e.g., 661F means that the camera points in the forward direction with viewing angle of about $66.1^{\circ}$, and 661A means that the camera points in the aft direction with a viewing angle of $66.1^{\circ}$. Pixels corresponding to viewing angle and sunlight direction within a $15^{\circ}$ cone are removed to avoid potential sunglint contamination.

Since most studies in the AirMSPI project focus on liquid water clouds, there are few cirrus cases in the AirMSPI dataset. The cirrus example for this study occurred at 21:20 UTC 28 January 2013 off the coast of Southern California at latitudes between $31.62^{\circ} \mathrm{N}$ and $31.68^{\circ} \mathrm{N}$ and at longitudes between $122.59^{\circ} \mathrm{W}$ and $122.68^{\circ} \mathrm{W}$ over the ocean A Red-Green-Blue (RGB) composite image is shown in Figure 4.

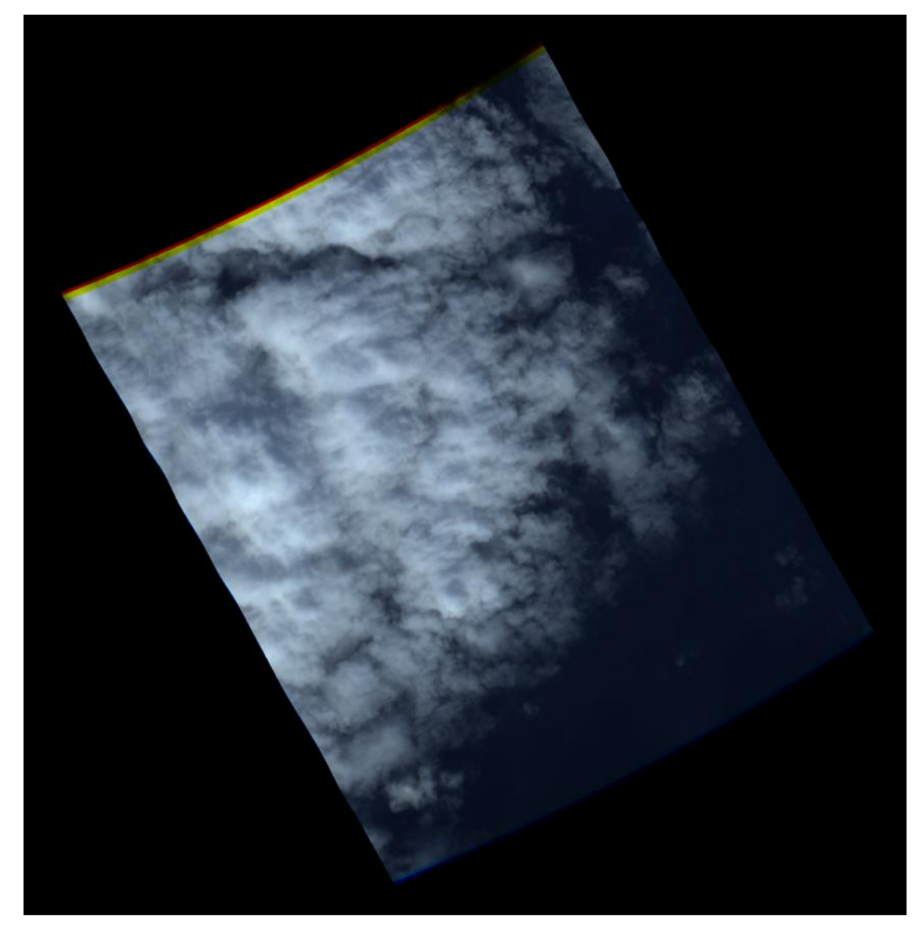

Figure 4. Red-Green-Blue (RGB) composite image (blue: $470 \mathrm{~nm}$, green: $555 \mathrm{~nm}$, and red: $660 \mathrm{~nm}$ ) with a pixel resolution of $10 \mathrm{~m}$ from the selected case in this study. The brightness is normalized to have better contrast for displaying photo details. 
Figure 5 shows the zenith angle and azimuth angle range of the sun and each AirMSPI camera. The azimuth angle is plotted in degrees clockwise from north. The vectors of both the solar azimuth angle and viewing azimuth angle in AirMSPI are defined as pointing in the direction of photon travel. To better depict the relation between sun location and viewing geometry, the plotted angle is the solar azimuth angle plus $180^{\circ}$. This treatment is for the solar azimuth angle only, not for the viewing azimuth angle. The radial distances reflect the zenith angle (solar zenith angle or viewing zenith angle). The color map plots the scattering angle for each pixel. The scattering angle is the propagation direction of the incident light after being scattered by ice particles to the AirMSPI camera. The aft cameras have larger scattering angles closer to backscattering than the forward cameras, because the aircraft flew toward the WNW in this data sample. The scattering angle is smaller for a forward camera with a high viewing zenith angle. However, the ranges of scattering angles overlap for each aft camera, and most aft camera scattering angles are between $130^{\circ}$ and $150^{\circ}$. For this case, the total reflectivity from the $661 \mathrm{~A}$ camera is much higher than from other cameras, which leads to an extremely high optical thickness in the retrieval process. To avoid potential biases, the 661A camera is not considered in the computation process. We did not see this feature in other granules, so it is less likely due to the calibration. Potential reasons are discussed in Section 4.3.
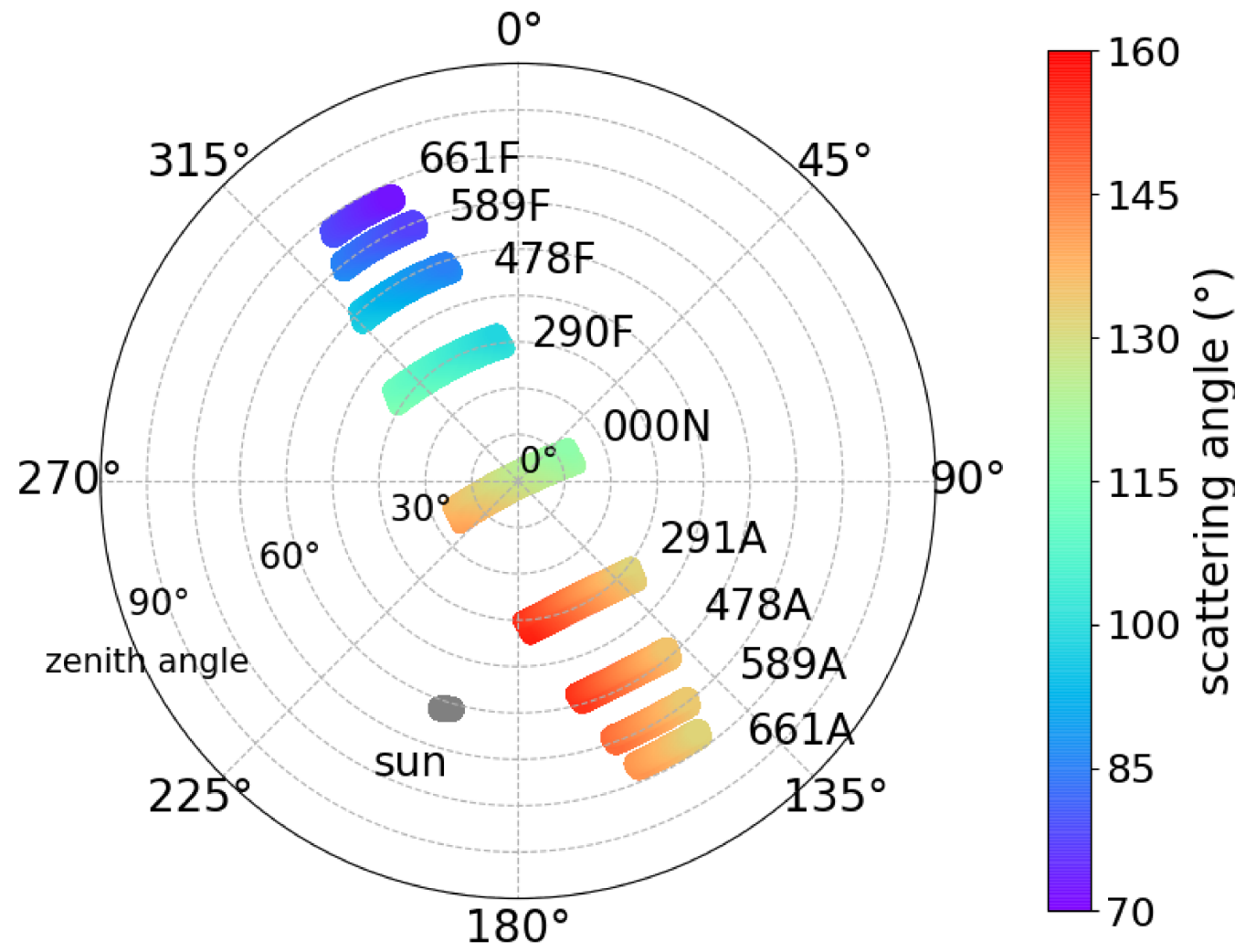

Figure 5. The geometry angle range of the sun and AirMSPI camera views in the selected case in this study. The azimuth angle range (solar azimuth angle plus $180^{\circ}$ or viewing azimuth angle) and zenith angle (solar zenith angle or viewing zenith angle) are plotted clockwise from the north $\left(0^{\circ}\right)$ and radial distance (angle from vertical), respectively. The color bar shows the solar scattering angle for each pixel.

\subsection{Inference of Ice Particle Shape}

We now apply the method proposed in Section 2.2 to infer the optimal ice habit model. The optimal inferred ice particle shape produced by each camera selection is shown in Figure 6 . The ice particle habits in Figure $6 \mathrm{a}, \mathrm{b}$ are retrieved using total reflectivity and polarized reflectivity, respectively. 


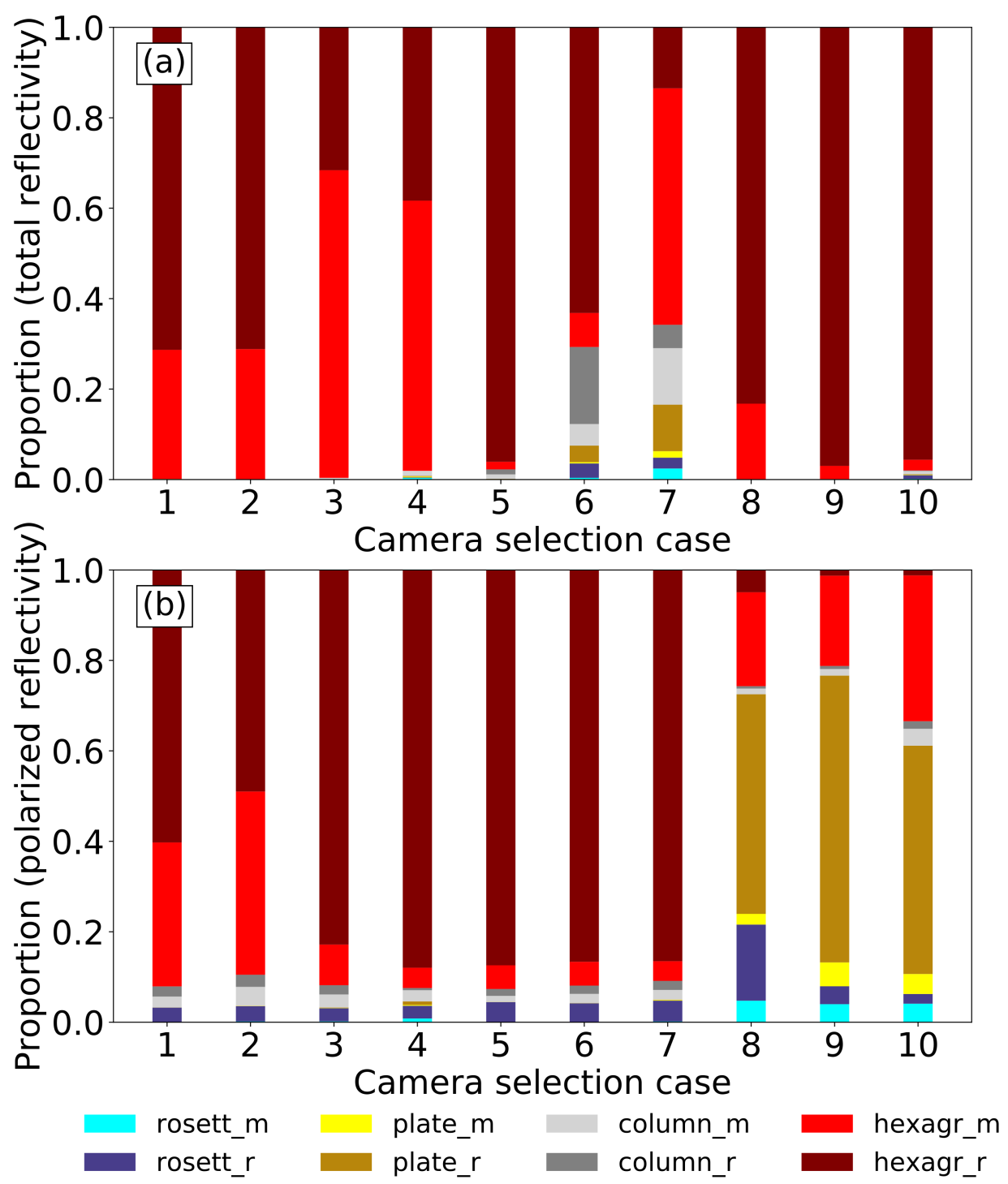

Figure 6. The proportion of inferred ice particle shapes in different camera selection cases using (a) total reflectivity and (b) polarized reflectivity.

As noted earlier, the viewing geometry angles are not the same for each camera. The selection of cameras is critical, because each camera produces a different retrieval $[7,29]$. To investigate the effect of camera selection on retrievals, Figure 6 shows retrievals from 10 different camera selection cases that are listed in Table 1 . The first four cases include all cameras, but we progressively removed the forward and aft pairs with the largest viewing angles. Since each pair of cameras has a different viewing zenith angle, these first four cases demonstrate the viewing zenith angle influence. The first case includes all cameras (except 661A), and the fourth case excludes all cameras with viewing angles $>30^{\circ}$. The other six cases focus on the influence of forward and aft camera selections. Cases 5, 6, and 7 use four, three, or two cameras up to 589F, and cases 8, 9, and 10 use four, three, or two cameras up to 589A. Case 5 includes the nadir camera and the forward cameras up to $589 \mathrm{~F}$, and case 8 uses the nadir camera and the aft cameras up to 589A. 
Table 1. The camera selection in each group. Cameras with a plus sign are included in a group, and those with a minus sign are not included.

\begin{tabular}{|c|c|c|c|c|c|c|c|c|c|}
\hline \multirow{2}{*}{ Case Index } & \multicolumn{9}{|c|}{ Camera Selection } \\
\hline & $661 F$ & $589 F$ & $478 \mathrm{~F}$ & $290 F$ & $000 N$ & 291A & $478 \mathrm{~A}$ & 589A & $661 A$ \\
\hline 1 & + & + & + & + & + & + & + & + & - \\
\hline 2 & - & + & + & + & + & + & + & + & - \\
\hline 3 & - & - & + & + & + & + & + & - & - \\
\hline 4 & - & - & - & + & + & + & - & - & - \\
\hline 5 & - & + & + & + & + & - & - & - & - \\
\hline 6 & - & + & + & + & - & - & - & - & - \\
\hline 7 & - & + & + & - & - & - & - & - & - \\
\hline 8 & - & - & - & - & + & + & + & + & - \\
\hline 9 & - & - & - & - & - & + & + & + & - \\
\hline 10 & - & - & - & - & - & - & + & + & - \\
\hline
\end{tabular}

When retrievals use total reflectivity (Figure 6a), the hexagonal column aggregate habit (either smooth or roughened particles) is chosen for the most pixels in all camera selection groups. The proportions of smooth particles in cases 3 and 4 are larger than in cases 1 and 2. Higher proportions of smooth particles are obtained in the retrievals when camera pairs with higher viewing zenith angles are excluded. Comparison of the retrievals between forward camera cases (cases 5-7) and aft camera cases (cases 8-10) indicates that the optimal ice particle habits are more diverse in forward camera cases (especially 6 and 7). From Case 5 to Case 7, as the nadir and 290F cameras are removed, the range of observed scattering angles also decreases, and the proportion of hexagonal aggregate habits decreases as well. Unlike the forward camera cases, the proportions of the selected ice models are quite stable among the three aft camera cases. While the number of cameras is different in each aft camera case, the range of scattering angles is similar in each case.

The retrievals using polarized reflectivity in this case study are shown in Figure 6b. Unlike the retrievals using total reflectivity, in the first four groups (with lower viewing angles), more ice pixels are best represented by the roughened hexagonal shape when the number of camera pairs is reduced. For the forward camera cases (5-7), the proportions of the various ice models selected for the pixels were almost identical in each group. The majority of the retrievals resulted in the selection of the roughened hexagonal particle in groups 3-7. The proportions of the optimal ice particle model selected in the aft camera cases (8-10) are significantly different than in the other seven cases. In the aft camera cases, the roughened plate was chosen for most of the pixels, but the variation of the optimal ice particle habit is high in every case. This is a result of the measured polarized reflectivity being significantly larger than the simulated polarized reflectivity in the nadir and aft cameras than in the forward cameras. Among those cameras, five models (smooth column, roughened column, smooth hexagonal, roughened hexagonal, and roughened bullet rosette shapes) provide similarly low polarized reflectivities. For aft cameras, the simulated polarized reflectivities with smooth and roughened plate models are both close to measured polarized reflectivities. This feature causes the plate habit to explain most pixels in aft camera selection cases but not in other selection groups in this study case.

The hexagonal aggregate column habit explains most pixels in both the total reflectivity and polarized reflectivity retrievals in this study case, as shown in Figure 6. This is consistent with a previous study [16] that was applied to ice clouds with optical thicknesses larger than 5 . Note that the roughened hexagonal aggregate model is also the ice model used in MODIS Collection 6. That implies the polarization properties of the MODIS Collection 6 model are consistent with the case in AirMSPI measurements.

The inconsistency $\left(s^{2}\right)$ between the simulated and measured reflectivity in each camera selection case is shown in Figure 7. The inconsistency in Figure 7a is defined as the standard deviation of retrieved optical thickness among selected cameras. The inconsistency is computed by Equations (4) and (5) for total reflectivity and polarized reflectivity, respectively. In 
Figure 7a, the inconsistency is smaller, with fewer pairs of cameras (compare cases 2 and 4), and aft camera cases (8-10) have a larger inconsistency than forward camera cases (5-7). The retrieved optical thickness values among forward cameras are low but much higher using aft cameras. Thus, the large inconsistency in Figure 7a is likely caused by retrieved high optical thickness values in aft cameras. The inconsistency in Figure $7 \mathrm{~b}$ using polarized reflectivity is also sensitive to the camera selection as in Figure 7a using total reflectivity. However, the differences in the polarized reflectivity have larger inconsistencies when forward cameras are involved.
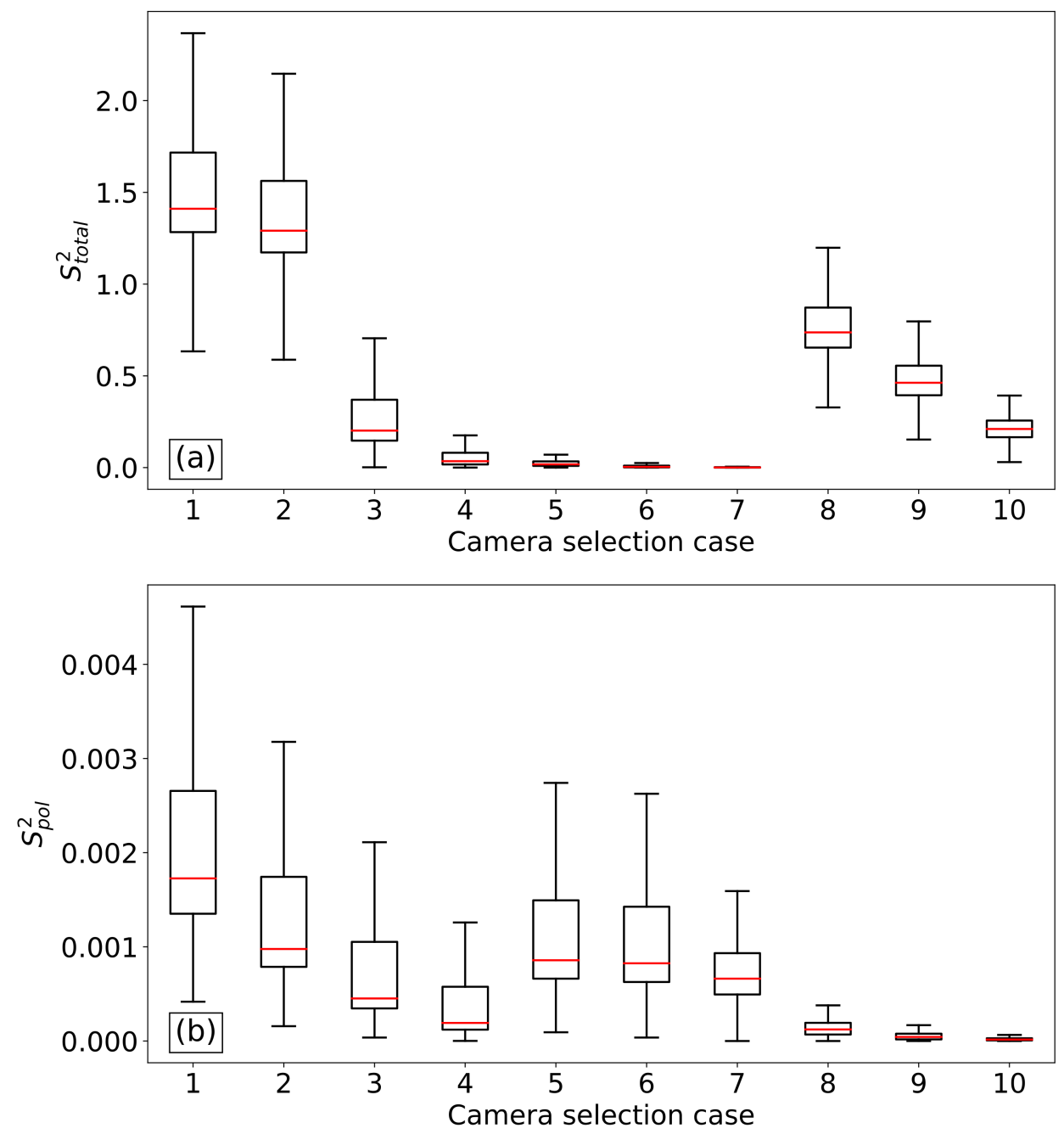

Figure 7. The inconsistency between model reflectivity with inferred ice particle models and measurements from (a) the total reflectivity and (b) polarized reflectivity for each different camera selection case.

The inconsistency in both total reflectivity and polarized reflectivity is reduced with fewer camera pairs with smaller viewing zenith angles (nearer to nadir). This means that the larger zenith angle camera measurements introduce larger inconsistency. A comparison of the forward and aft camera cases indicates that the inconsistency of forward camera cases is significantly higher than for aft camera cases in polarized reflectivity, but the inconsistency is lower in the total reflectivity. Note that the diversity of the optimal ice models is higher with the forward camera cases in Figure 6a but higher with the aft camera cases in Figure $6 \mathrm{~b}$. This indicates that the variation of the optimal ice models decreases when there is an inconsistency between simulations and measurements. 


\section{Discussions}

\subsection{Retrievals from Total Reflectivity}

The retrieval method based on total reflectivity in this study relies on the retrieved optical thickness from all cameras. We first examine the median value of retrieved optical thickness using total reflectivity obtained from multiple ice particle models for each camera (Figure 8). Specifically, the optical thickness value is retrieved for every pixel in conjunction with an individual ice particle model. For each camera, the median value is calculated from the individual pixel retrievals.

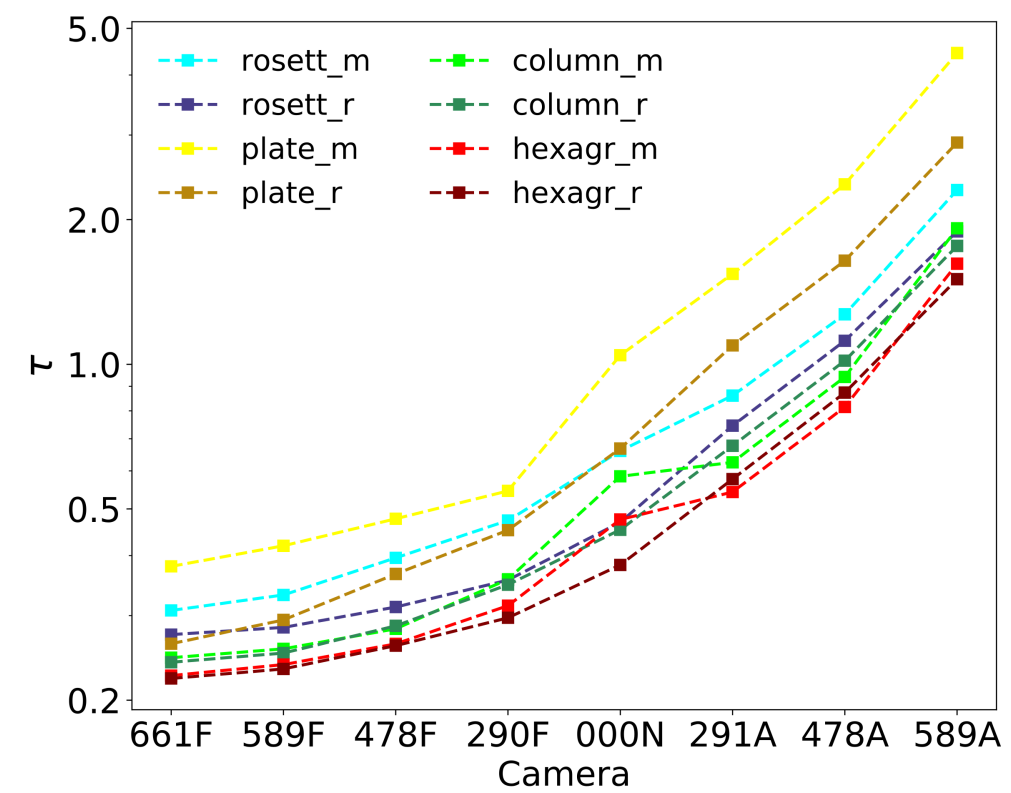

Figure 8. The median values of retrieved ice cloud optical thickness with different ice particle models using the total reflectivity from each camera. The smooth particle models are denoted with ("_m"), and the roughened models are denoted with ("_r").

As shown in Equation (4), the optical thickness is retrieved for the cloudy pixels and all selected cameras using the eight selected ice particle models. An optimal ice particle model has the least variation of optical thicknesses among selected cameras. Figure 8 shows that optical thickness values are significantly different from each camera in this data sample. For the nadir camera $(000 \mathrm{~N})$, the median values of optical thicknesses are around 0.5. With the forward and aft cameras, the median retrieved optical thickness values are lower and higher, respectively, than for the nadir camera. The optical thickness decreases with increasing the viewing zenith angle for the forward cameras but significantly increases with increasing viewing zenith angle for the aft cameras. It is interesting that we do not see the similar trends of increasing optical thickness with viewing zenith angle in both forward and aft cameras. Specifically, for each camera pair (a forward and aft camera with the same viewing zenith angle), the optical thickness from the aft camera is significantly higher than from the forward camera. For each camera pair, the zenith angles are the same, but the azimuth angles are not.

By comparing the optical properties retrieved by assuming roughened or smooth ice particles as shown in Figure 8, the roughened models tend to provide higher optical thickness values than the corresponding smooth models for most habits. Specifically, the plate model provides the highest optical thickness, and the hexagonal aggregate model provides the lowest optical thickness values among all selected models.

Another important factor that affects retrievals is which scattering angles are sampled. To investigate the influence of viewing geometry angles on satellite retrievals, Figure 9 shows the retrieved optical thickness as a function of the scattering angle. The optical thickness is the mean value of retrievals from all eight ice particle models. In other words, 
each pixel has eight retrieved optical thicknesses by applying different ice particle models, and the median value of these eight optical thickness is calculated and plotted in this figure. The frequency versus scattering angle is also provided by using the colormap and the boxplot in this figure.

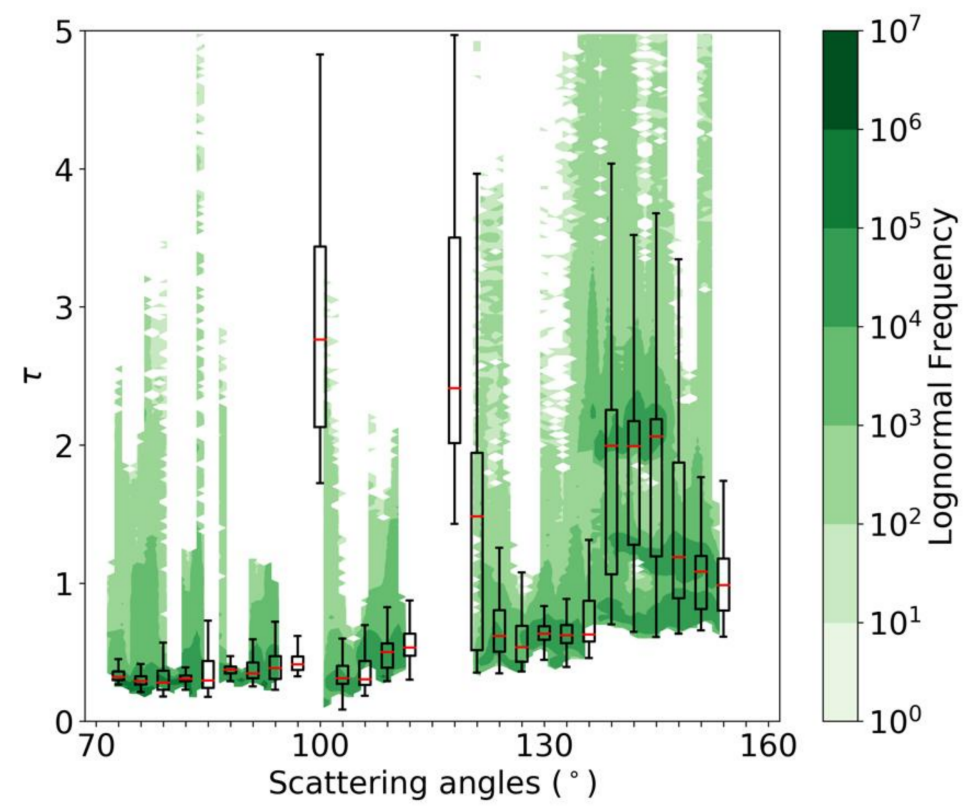

Figure 9. The retrieved optical thickness values using total reflectivity as a function of the scattering angle. The colormap demonstrates the pixel density.

Figure 9 shows that only scattering angles between $70^{\circ}$ and $160^{\circ}$ are sampled in this study. The relationship between the camera and scattering angles can be found in Figure 5 . The optical thicknesses between the scattering angles of $120^{\circ}$ and $140^{\circ}$ are retrieved from the $000 \mathrm{~N}$ camera and between the scattering angles of $100^{\circ}$ and $110^{\circ}$ are from the $290 \mathrm{~F}$ camera. At scattering angles less than $100^{\circ}$, optical thickness is retrieved from the $661 \mathrm{~F}$, $589 \mathrm{~F}$, and $478 \mathrm{~F}$ cameras, and their values are similar.

The results show that the retrieved optical thickness value roughly increases with increasing scattering angle. The optical thickness values are higher at backscattering angles between $130^{\circ}$ and $150^{\circ}$ than at other scattering angles. At backscattering angles between about $140^{\circ}$ and $150^{\circ}$, the retrieved optical thickness values with the highest frequencies occur at approximately $0.5,1$, and 2, which, interestingly, are obtained using the 291A, 478A, and 589A cameras. These three cameras (291A, 478A, and 589A) observe the pixels at similar scattering angles and basically the same solar zenith angle. The interesting point is that we did not see significant differences in the retrieved optical thickness values from other cameras, especially from forward cameras. This feature indicates that the 3D radiative effect may contribute to the different retrievals.

\subsection{Retrievals from Polarized Reflectivity}

Figure 10 is similar to Figure 8 but shows the median value of simulated polarized reflectivities. Each simulated polarized reflectivity is computed by assuming the optical thickness retrieved from total reflectivity for the ice model, as explained in Section 2.2. All eight ice particle models are applied to simulate polarized reflectivities and displayed separately. The median value of measured polarized reflectivity is also plotted in the same figure. 


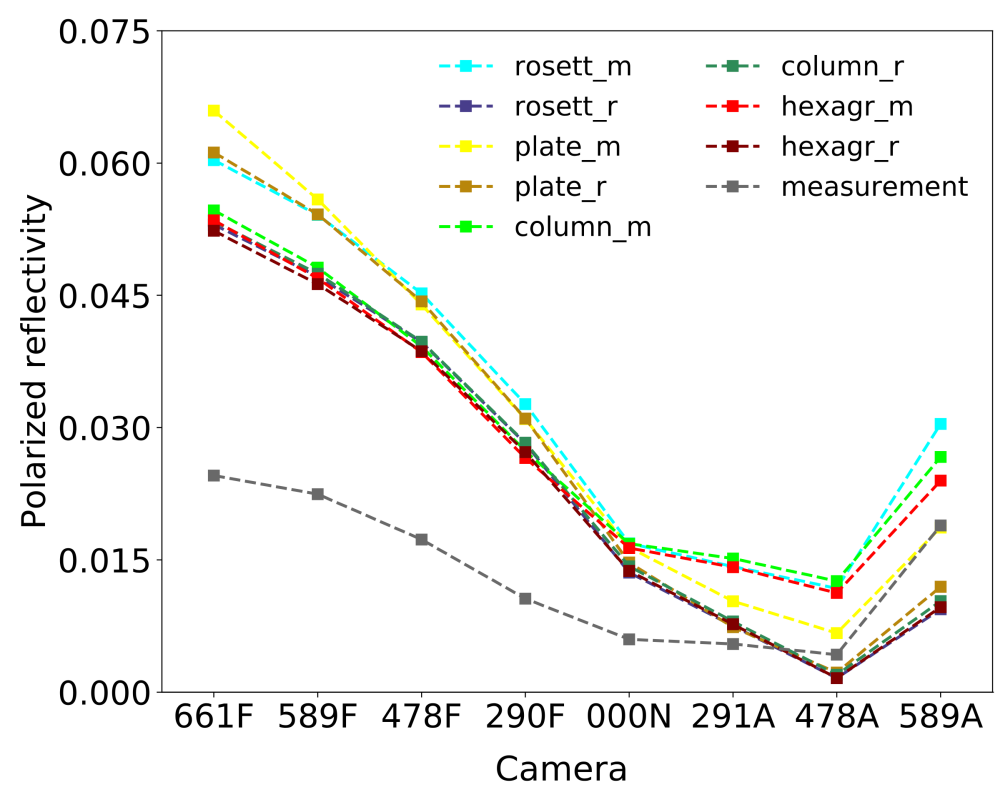

Figure 10. The median values of the model simulated polarized reflectivity with different ice particle models and the measured polarized reflectivity from each camera. The smooth particle models are denoted with ("_m") and the roughened models are denoted with ("_r").

Figure 10 shows that the simulated polarized reflectivity in all models is higher than the measurements for most of the cameras. The differences between model polarized reflectivity and measured reflectivity are significantly larger using forward cameras than aft cameras, especially with forward large zenith angle cameras. The simulated reflectivities for the plates are closest to the measured polarized reflectivities using the $478 \mathrm{~A}$ and 589A cameras.

In comparing each ice particle habit, the roughened model has a lower simulated polarized reflectivity than the smooth model. For most cameras, the roughened hexagonal aggregate model provides the lowest simulated polarized reflectivity values for most cameras. The differences among polarized reflectivities are larger for high zenith angle than low zenith angle cameras. Note that the absolute value of the retrieved optical thickness does not directly impact the determination of the optimal ice particle models for both retrieval methods in this study, only the standard deviations among all cameras.

The polarized reflectivity differences as a function of scattering angle are shown in Figure 11. The ordinate is the mean value of the simulated polarized reflectivity minus the measured polarized reflectivity for each of the eight ice particle models. The polarized reflectivity difference decreases with increasing scattering angle. The large differences illustrate that polarized reflectivity simulations need improvement at the forward scattering angles.

Unlike the case shown in Figure 9, there are no obvious multiple frequency peaks in the optical thickness values in the backscattering directions in Figure 11. This indicates that the polarized reflectivity simulations for cirrus may be less sensitive to viewing angles in different cameras than to the total reflectivity in backscattering directions.

\subsection{Uncertainties, Limitations, and Perspectives}

While our goal is to find a model that fits all observations, it is very difficult for multiple reasons. First of all, ice cloud particles have unique shapes that are mostly quite complex. Ice particles form through either homogeneous or heterogeneous ice nucleation and then grow/decay with time. Different form types depend on many factors, such as ice nucleating particle existence, temperature, supersaturation, updraft strength, etc. [30]. The homogeneous-dominated cirrus is more abundant during the winter at midlatitudes and over mountainous terrain [31-33]. Secondly, using a single ice particle habit 
to represent homogeneous-dominated cirrus clouds is a challenge. Even for the simple plate column, there are multiple variables that affect the shape, such as particle size, aspect ratio, degree of surface roughness, and crystal orientation. It may be unrealistic to expect that a single model could adequately represent all real ice crystal shapes, but for global satellite retrievals, this is basically how the data reduction process works due, in part, to computational limitations. For example, the number of dimensions must be reduced when building a look-up table of single scattering properties to make global retrievals tractable. For the 865-nm channel, one example is to choose a single effective particle size for the ice particle model, because it does not significantly affect the retrievals, as discussed in Section 2.1.

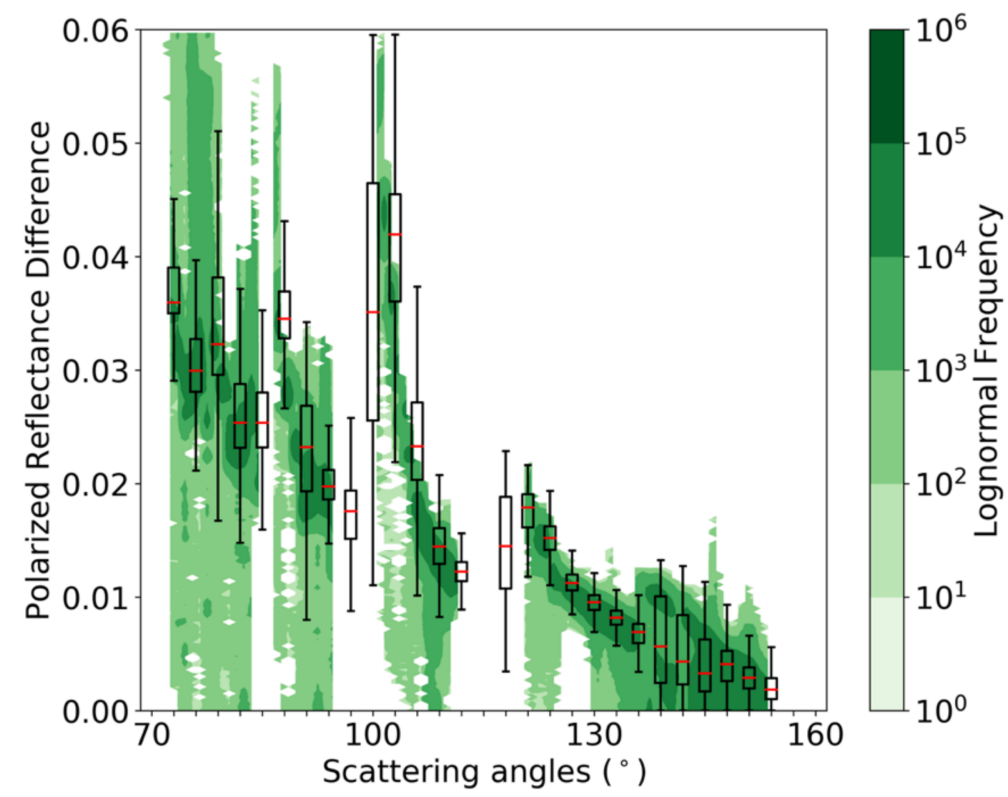

Figure 11. The same as Figure 9 but for the polarized reflectivity difference. The polarized difference is the mean value of 8 simulated polarized reflectivities assuming retrieved optical thickness values based on the total reflectivity minus the measured polarized reflectivity computed with different ice models.

Other reasons could also contribute to the discrepancies between modeled and realworld ice particles. Several studies focused on crystal orientation and compared them to randomly oriented ice crystals, which are assumed in this study. Oriented (particularly horizontally oriented) ice crystals provide very different single-scattering properties, especially for certain optical phenomena such as parhelia (sundogs) [34]. The possibility of oriented ice crystals being present increases when the updraft velocities are very low and could be associated with very high radiance observed by the 661A camera. 3D radiative transfer effects could offer another potential reason.

Some limitations are also discussed here. Mixed-phase clouds with both ice and liquid phase particles are always a challenge for the remote sensing of clouds. The common approach is to set a very low temperature as a threshold to filter out mixed phase clouds. Due to limited data, this proof-of-concept study has not applied this filter to the retrievals. However, considering that only a small part of pixels are identified as liquid water in this data sample, mixed-phase clouds should not significantly affect the retrievals.

The scattering angle selection is very important in the retrieval algorithm. The scattering angles in this single case study are between $70^{\circ}$ and $160^{\circ}$. Observations at scattering angles less than $70^{\circ}$ (forward scattering) are not generally available for most satellite and aircraft observations. One reason is because the sensors need to be protected from observing direct sunlight. 
The scattering viewing geometries could also affect the application range of the results from this data sample. For example, the scattering angle observed by a camera strongly affects the retrieved particle shape model. The flight heading azimuth is $150^{\circ}$ in this study case, which means the flight direction was perpendicular to the sun position at the measurement time. The forward and aft cameras impact the particle shape retrievals somewhat similarly. Therefore, the results such as the zenith angle dependence may change when applying this method to larger datasets.

Another limitation is the look-up table retrieval technique. The computation of the look-up table usually requires several physical assumptions to minimize computation resources, which causes the parameter retrieval to be computed by interpolating the lookup table. Due to that, researchers tend to put much effort into minimizing the degradation in the retrieval precision. In the future, artificial intelligence deep learning techniques may more successfully interpret complex scenes, even if such situations are currently simply rejected because they cannot be satisfactorily modeled for inclusion in look-up tables.

Compared to cross-track scanning sensors, the advantage of a multi-angle imaging polarimeter is to provide multiple viewing angles of each pixel and to observe the degree of linear polarization. Different viewing zenith angle cameras provide measurements that capture more cloud scattering information, including both upward and oblique radiances. This provides an unprecedented understanding of the cloud 3D radiative transfer effect. For example, detected radiances at nadir for overhead sun are likely smaller than radiative transfer simulations due to horizontal leakage of radiation from cloud sides, especially in horizontally heterogeneous cloud fields. However, by using multiple angle radiances in satellite retrievals, cloud properties such as optical thickness and particle size can be retrieved in conjunction with multiple viewing configurations. By implicitly incorporating cloud 3D effects, the improved cloud properties should result in a better knowledge of factors affecting the global energy balance.

\section{Summary and Conclusions}

In this study, we developed a remote sensing technique to infer an optimal ice particle model for cirrus using multidirectional polarimetric observations at $865 \mathrm{~nm}$. As part of the retrieval process, the optical thickness retrieved from total reflectivity is applied to the computation of polarized reflectivity. This retrieval approach provides better consistency for cirrus retrievals based on the limited data for this proof-of-concept study. The optical thickness for cirrus is less than 3-5 where multiple scattering does not dominate, and globally, about $30 \%$ of clouds are cirrus. We avoid the common treatment of assuming the ice cloud optical thickness to be larger than 5 for inferring the optimal ice particle shape model and extend the methodology to an optical thickness less than 5 . We apply this method to Airborne Multi-angle SpectroPolarimeter Imager (AirMSPI) measurements to infer optimal ice particle models for cirrus clouds as a proof-of-concept study, including assessing the influence of different camera selections on the resulting optimal ice particle model from the total reflectivity and polarized reflectivity.

The hexagonal aggregate column habit (with either smooth or roughened surfaces) explains most pixels in both the total reflectivity and polarized reflectivity retrievals in this study case. The optimal ice model in different camera selection groups shows that zenith angle dependence (cameras viewing closer to or farther from nadir) has less effect on ice particle shape retrievals than selecting forward or aft viewing cameras. The inferred ice particle habit does not change much as the number of selected cameras increases, but a smooth particle model works for more pixels in total reflectivity retrievals, and a roughened particle model works better for polarized reflectivity retrievals. However, when reducing the number of selected camera pairs, or with fewer cameras, the inconsistencies between the simulated and measured ice cloud reflectivities are significantly decreased. The smallest inconsistency exists using forward cameras in the total reflectivity retrievals or using aft cameras in the polarized reflectivity retrievals. However, the pixels with the smallest inconsistencies also have the largest diversity of the optimal inferred ice model. These 
findings point to the fact that a large inconsistency of polarization properties exists when forward cameras (i.e., scattering angles between $70^{\circ}$ and $100^{\circ}$ ) are involved in this data sample. Since retrievals are sensitive to the scattering angle, the results about ice particle shape retrieval and camera selection from this data sample may change with different scattering viewing geometries or larger datasets.

As an airborne instrument, AirMSPI provides higher resolution data than satellite sensors for the multi-angle imaging measurements. It is very useful to infer the optimal ice particle models for cirrus clouds using such fine-resolution polarized measurements. However, since the AirMSPI project is not mainly focused on ice clouds, the observations that contain cirrus clouds are rare. Therefore, future research should apply this technique to large volume datasets, like POLDER, to further evaluate the effect of camera selection (more precisely, scattering angle selection) on retrievals and to seek a particle optical property model that is more consistent for a wider range of observation geometries.

Author Contributions: Conceptualization, Y.W. and P.Y.; methodology, Y.W. and P.Y.; software, Y.W.; data curation, Y.W.; writing—original draft preparation, Y.W.; writing-review and editing, P.Y., M.D.K. and B.A.B.; visualization, Y.W.; supervision, P.Y., B.A.B. and M.D.K.; project administration, P.Y.; and funding acquisition, P.Y. All authors have read and agreed to the published version of the manuscript.

Funding: This research was partly supported by endowment funds related to the David Bullock Harris Chair in Geosciences at the College of Geosciences, Texas A\&M University (account number 02-512231-10000).

Institutional Review Board Statement: Not applicable.

Informed Consent Statement: Not applicable.

Data Availability Statement: These AirMSPI data were obtained from the NASA Langley Research Center Atmospheric Science Data Center (https: / / earthdata.nasa.gov / eosdis/ daacs/asdc).

Acknowledgments: These data were obtained from the NASA Langley Research Center Atmospheric Science Data Center. Portions of this research were conducted with the advanced computing resources provided by Texas A\&M High Performance Research Computing.

Conflicts of Interest: The authors declare no conflict of interest.

\section{References}

1. Mishchenko, M.I.; Rossow, W.B.; Macke, A.; Lacis, A.A. Sensitivity of cirrus cloud albedo, bidirectional reflectance and optical thickness retrieval accuracy to ice particle shape. J. Geophys. Res. Atmos. 1996, 101, 16973-16985. [CrossRef]

2. Baran, A.J. A review of the light scattering properties of cirrus. J. Quant. Spectrosc. Radiat. Transf. 2009, 110, 1239-1260. [CrossRef]

3. Yang, P.; Liou, K.N.; Bi, L.; Liu, C.; Yi, B.Q.; Baum, B.A. On the radiative properties of ice clouds: Light scattering, remote sensing, and radiation parameterization. Adv. Atmos. Sci. 2015, 32, 32-63. [CrossRef]

4. Loeb, N.G.; Yang, P.; Rose, F.G.; Hong, G.; Sun-Mack, S.; Minnis, P.; Kato, S.; Ham, S.-H.; Smith, W.L., Jr.; Hioki, S. Impact of ice cloud microphysics on satellite cloud retrievals and broadband flux radiative transfer model calculations. J. Clim. 2018, 31, 1851-1864. [CrossRef]

5. Deschamps, P.-Y.; Bréon, F.-M.; Leroy, M.; Podaire, A.; Bricaud, A.; Buriez, J.-C.; Seze, G. The POLDER mission: Instrument characteristics and scientific objectives. IEEE Trans. Geosci. Remote Sens. 1994, 32, 598-615. [CrossRef]

6. Diner, D.J.; Beckert, J.C.; Reilly, T.H.; Bruegge, C.J.; Conel, J.E.; Kahn, R.A.; Martonchik, J.V.; Ackerman, T.P.; Davies, R.; Gerstl, S.A. Multi-angle Imaging SpectroRadiometer (MISR) instrument description and experiment overview. IEEE Trans. Geosci. Remote Sens. 1998, 36, 1072-1087. [CrossRef]

7. Wang, Y.; Yang, P.; Hioki, S.; King, M.D.; Baum, B.A.; Di Girolamo, L.; Fu, D. Ice cloud optical thickness, effective radius, and ice water path inferred from fused MISR and MODIS measurements based on a pixel-level optimal ice particle roughness model. J. Geophys. Res. Atmos. 2019, 124, 12126-12140. [CrossRef]

8. Doutriaux-Boucher, M.; Buriez, J.C.; Brogniez, G.; C.-Labonnote, L.; Baran, A.J. Sensitivity of retrieved POLDER directional cloud optical thickness to various ice particle models. Geophys. Res. Lett. 2000, 27, 109-112. [CrossRef]

9. McFarlane, S.A.; Marchand, R.T.; Ackerman, T.P. Retrieval of cloud phase and crystal habit from Multiangle Imaging Spectroradiometer (MISR) and Moderate Resolution Imaging Spectroradiometer (MODIS) data. J. Geophys. Res. Atmos. 2005, 110. [CrossRef]

10. Xie, Y.; Yang, P.; Kattawar, G.W.; Minnis, P.; Hu, Y.; Wu, D.L. Determination of ice cloud models using MODIS and MISR data. Int. J. Remote Sens. 2012, 33, 4219-4253. [CrossRef]

11. Wang, Y.; Hioki, S.; Yang, P.; King, M.D.; Di Girolamo, L.; Fu, D.; Baum, B.A. Inference of an optimal ice particle model through latitudinal analysis of MISR and MODIS data. Remote Sens. 2018, 10, 1981. [CrossRef] 
12. Chepfer, H.; Minnis, P.; Young, D.; Nguyen, L.; Arduini, R.F. Estimation of cirrus cloud effective ice crystal shapes using visible reflectances from dual-satellite measurements. J. Geophys. Res. Atmos. 2002, 107. [CrossRef]

13. Sun, W.; Loeb, N.G.; Yang, P. On the retrieval of ice cloud particle shapes from POLDER measurements. J. Quant. Spectrosc. Radiat. Transf. 2006, 101, 435-447. [CrossRef]

14. Baran, A.; Labonnote, L.C. A self-consistent scattering model for cirrus. I: The solar region. J. Quant. Spectrosc. Radiat. Transf. 2007, 133, 1899-1912. [CrossRef]

15. Van Diedenhoven, B.; Cairns, B.; Geogdzhayev, I.; Fridlind, A.; Ackerman, A.; Yang, P.; Baum, B.A. Remote sensing of ice crystal asymmetry parameter using multi-directional polarization measurements-Part 1: Methodology and evaluation with simulated measurements. Atmos. Meas. Tech. 2012, 5, 2361-2374. [CrossRef]

16. Cole, B.; Yang, P.; Baum, B.A.; Riedi, J.; C-Labonnote, L. Ice particle habit and surface roughness derived from PARASOL polarization measurements. Atmos. Chem. Phys. 2014, 14, 3739-3750. [CrossRef]

17. Hioki, S.; Yang, P.; Baum, B.A.; Platnick, S.; Meyer, K.G.; King, M.D.; Riedi, J. Degree of ice particle surface roughness inferred from polarimetric observations. Atmos. Chem. Phys. 2016, 16, 7545-7558. [CrossRef]

18. Masuda, K.; Takashima, T. Feasibility study of derivation of cirrus information using polarimetric measurements from satellite. Remote Sens. Environ. 1992, 39, 45-59. [CrossRef]

19. Diner, D.J.; Xu, F.; Garay, M.J.; Martonchik, J.V.; Rheingans, B.E.; Geier, S.; Davis, A.; Hancock, B.; Jovanovic, V.; Bull, M. The Airborne Multiangle SpectroPolarimetric Imager (AirMSPI): A new tool for aerosol and cloud remote sensing. Atmos. Meas. Tech. 2013, 6, 2007-2025. [CrossRef]

20. McFarlane, S.A.; Marchand, R.T. Analysis of ice crystal habits derived from MISR and MODIS observations over the ARM Southern Great Plains site. J. Geophys. Res. Atmos. 2008, 113. [CrossRef]

21. Zhang, Z.; Yang, P.; Kattawar, G.; Riedi, J.; Labonnote, L.; Baum, B.A.; Platnick, S.; Huang, H.-L. Influence of ice particle model on satellite ice cloud retrieval: Lessons learned from MODIS and POLDER cloud product comparison. Atmos. Chem. Phys. 2009, 9, 7115-7129. [CrossRef]

22. Yang, P.; Bi, L.; Baum, B.A.; Liou, K.-N.; Kattawar, G.W.; Mishchenko, M.I.; Cole, B. Spectrally consistent scattering, absorption, and polarization properties of atmospheric ice crystals at wavelengths from 0.2 to $100 \mu \mathrm{m}$. J. Atmos. Sci. 2013, 70, 330-347. [CrossRef]

23. Yang, P.; Liou, K. Single-scattering properties of complex ice crystals in terrestrial atmosphere. Beitr. Phys. Atmosphare-Contrib. Atmos. Phys. 1998, 71, 223-248.

24. Platnick, S.; Meyer, K.G.; King, M.D.; Wind, G.; Amarasinghe, N.; Marchant, B.; Arnold, G.T.; Zhang, Z.; Hubanks, P.A.; Holz, R.E.; et al. The MODIS cloud optical and microphysical products: Collection 6 updates and examples from Terra and Aqua. IEEE Trans. Geosci. Remote Sens. 2016, 55, 502-525. [CrossRef] [PubMed]

25. Yang, P.; Hioki, S.; Saito, M.; Kuo, C.P.; Baum, B.A.; Liou, K.-N. A review of ice cloud optical property models for passive satellite remote sensing. Atmosphere 2018, 9, 499. [CrossRef]

26. Nakajima, T.; King, M.D. Determination of the optical thickness and effective particle radius of clouds from reflected solar radiation measurements. Part I: Theory. J. Atmos. Sci. 1990, 47, 1878-1893. [CrossRef]

27. Huang, X.; Yang, P.; Kattawar, G.; Liou, K.-N. Effect of mineral dust aerosol aspect ratio on polarized reflectance. J. Quant. Spectrosc. Radiat. Transf. 2015, 151, 97-109. [CrossRef]

28. Cox, C.; Munk, W. Measurement of the roughness of the sea surface from photographs of the sun's glitter. J. Opt. Soc. Am. 1954, 44, 838-850. [CrossRef]

29. Liang, L.; Girolamo, L.D. A global analysis on the view-angle dependence of plane-parallel oceanic liquid water cloud optical thickness using data synergy from MISR and MODIS. J. Geophys. Res. Atmos. 2013, 118, 2389-2403. [CrossRef]

30. Mitchell, D.L.; Mejia, J.; Garnier, A.; Tomii, Y.; Krämer, M.; Hosseinpour, F. An estimate of global, regional and seasonal cirrus cloud radiative effects contributed by homogeneous ice nucleation. Atmos. Chem. Phys. Discuss. 2020. [CrossRef]

31. Mitchell, D.L.; Garnier, A.; Pelon, J.; Erfani, E. CALIPSO (IIR-CALIOP) retrievals of cirrus cloud ice-particle concentrations. Atmos. Chem. Phys. 2018, 18, 17325-17354. [CrossRef]

32. Sourdeval, O.; Gryspeerdt, E.; Krämer, M.; Goren, T.; Delanoë, J.; Afchine, A.; Hemmer, F.; Quaas, J. Ice crystal number concentration estimates from lidar-radar satellite remote sensing-Part 1: Method and evaluation. Atmos. Chem. Phys. 2018, 18, 14327-14350. [CrossRef]

33. Gryspeerdt, E.; Sourdeval, O.; Quaas, J.; Delanoë, J.; Krämer, M.; Kühne, P. Ice crystal number concentration estimates from lidar-radar satellite remote sensing-Part 2: Controls on the ice crystal number concentration. Atmos. Chem. Phys. 2018, 18, 14351-14370. [CrossRef]

34. Saito, M.; Yang, P. Oriented ice crystals: A single-scattering property database for applications to lidar and optical phenomenon simulations. J. Atmos. Sci. 2019, 76, 2635-2652. [CrossRef] 